\title{
Endocrine disrupting compounds (EDCs) in environmental matrices: review of analytical strategies for pharmaceuticals, estrogenic hormones, and alkylphenol compounds
}

\begin{abstract}
Endocrine disrupting compounds (EDCs) have been widely reported as potential carcinogenic threats to the human population. The release of EDCs to environmental compartments, such as water, sediment, and biota, has been monitored extensively. Considering the typically low levels of EDC concentrations found in environmental samples and the complexity of biota matrices, the main challenge is with the extraction and cleanup of samples, as well as with finding a sensitive enough instrumentation system for analyte detection. This paper presents a review of recent trends in the analysis of EDCs in environmental matrices. The focus of this review is three classes of environmentally important EDCs; namely, pharmaceuticals, estrogenic hormones, and alkylphenol compounds. Discussions about state-of-the-art instrumentation and sample preparation techniques, as well as a review of sample storage and preservation, are highlighted. Overall, the use of LC-MS-MS as an instrumentation technique has increased over the past 15 years.
\end{abstract}

Keyword: Endocrine disrupting compounds (EDCs); Pharmaceuticals; Estrogenic hormones; Alkylphenol; Environmental matrices; Biota; Sample preservation 